For reprint orders, please contact: reprints@futuremedicine.com

\title{
Why hypofractionate stereotactic radiosurgery for brain metastases?
}

\author{
"Stereotactic radiosurgery is a standard of care therapy for patients with \\ limited brain metastases."
}

\author{
Sten Myrehaug ${ }^{*, 1}$, Hany Soliman', Chia-Lin Tseng', Mark Ruschin², \\ David Larson ${ }^{3} \&$ Arjun Sahgal $^{1}$
}

First draft submitted: 27 May 2016; Accepted for publication: 6 June 2016;

Published online: 17 June 2016

Lars Leksell redefined the concept of radiotherapy to focal intracranial pathology, with his publication in 1951 'The Stereotaxic Method and Radiosurgery of the Brain' [1]. Initially conceived for functional disorders, his method introduced the world to the concept of stereotactic radiosurgery (SRS) based on a single high dose of radiation delivered with the patient in a rigid head immobilization system that yielded the stereotactic coordinates for intracranial target localization. Eventually, the technology morphed into the Gamma Knife (Elekta AB, Stockholm, Sweden) with 201 individual cobalt beams converging to the isocenter generating a high precision 'shot' of radiation. In later years, SRS was applied to the treatment of brain metastases as it became possible to merge multiple shots of radiation to cover the intended target as visualized first on CT, and then MRI. Single fraction SRS for brain metastases was considered a major departure from the traditional practice of whole brain radiation (WBRT), and several randomized trials ensued comparing WBRT to WBRT plus SRS, and then SRS alone to WBRT plus SRS [2-5]. We have now learned as a result of these trials, and large-scale analyses [6,7] that SRS is a standard of care therapy for patients with limited brain metastases. This is due to both a more favorable side effect profile as compared with WBRT and a survival advantage that may be observed in selected patients $[4,8]$.

The maximum tolerated dose of single fraction radiosurgery was established by the RTOG 90-05 clinical trial [9]. Stratification was based on tumor size with acute toxicity as primary end point. This practice defining study determined that as tumors increased in diameter, the radiation dose must be de-escalated to respect the risk of serious toxicity. As a result, tumors $\leq 2 \mathrm{~cm}$ have been treated largely with $20-24 \mathrm{~Gy}, 2.1-3.0 \mathrm{~cm}$ with 18 Gy and 3.1-4.0 cm 15 Gy. Tumors larger than $4.0 \mathrm{~cm}$ have often been considered ineligible for SRS due to a prohibitive adverse event profile. The relationship between volume of brain tissue irradiated to a high single dose and risk of radiation necrosis ( $\mathrm{RN}$ ) became clear as parameters such as the volume of targeted tissue receiving 12 Gy (V12Gy) and 10 Gy (V10Gy) were shown to be

\section{KEYWORDS}

- brain metastases

- hypofractionation - stereotactic radiosurgery

\section{"However, the rates of local control have suffered for tumors $>2 \mathrm{~cm}$ due to the necessary dose de-escalation.”}




\section{"Modern radiation oncology and stereotactic radiosurgery apparatus have adapted to support the growing trend of hypofractionated stereotactic radiosurgery.”}

predictive [10]. As a result, modern day SRS has been deemed safe when not exceeding the RTOG 90-05 dose guidelines with a predictable adverse event profile. We typically quote rates of symptomatic necrosis ranging from 5 to $10 \%$. However, the rates of local control have suffered for tumors $>2 \mathrm{~cm}$ due to the necessary dose de-escalation. One of the early studies of single fraction SRS that observed this relationship reported a 12 -month local control rate of $45 \%$ for tumors $>2 \mathrm{~cm}$ versus $85 \%$ when $\leq 2 \mathrm{~cm}$ [11].

Initial strategies to improve upon local tumor control consisted mainly of the addition of WBRT. It was demonstrated that local control is improved; however, at the expense of a greater risk of $\mathrm{RN}$, neurocognitive damage and deterioration in QoL $[4,8]$. Therefore, an alternative focal strategy was in need. With the development of image-guided linac-based radiotherapy incorporating multi-leaf collimators, intensity modulation and arc-based radiation, robotic six degree-of-freedom positioning corrections and the use of relocatable noninvasive head frames, millimeter accuracy can now be achieved without an invasive frame based apparatus [12]. The field of hypofractionated radiosurgery began.

Hypofractionation exploits the biologic advantages of fractionation (normal tissue repair, redistribution of cells to a more sensitive phase of the cell cycle and reoxygenation), while escalating the total dose in a short period of time to overcome any repopulation [13]. As such most deliver hypofractionated SRS in 2-5 fractions and a dose per fraction of at least 5 Gy. These high doses per fraction may also capitalize on activation of the ceramide pathway which has been shown to trigger tumor vascular endothelial apoptosis which otherwise would not be activated at standard 1.8-3.0 Gy per day [14]. This approach was initially for tumors greater than $3-4 \mathrm{~cm}$ and is now applied in our center (Sunnybrook Odette Cancer Centre, University of Toronto) for most tumors $>2 \mathrm{~cm}$ as a means to maximize local control while maintaining acceptable rates of RN.

Several single institution series have reported hypofractionated SRS results showing trends toward improved or equivalent local control rates with less rates of $\mathrm{RN}$ despite the larger tumor volumes irradiated. For example, Feuvret et al. [15] compared single fraction SRS to hypofractionated SRS (7.7 Gy $\times 3$ fractions) for large brain metastases (median diameter $3.7 \mathrm{~cm}$ vs $4.4 \mathrm{~cm}$, respectively). Despite the larger median size of tumor treated, compared with single fraction treatment, hypofractionation demonstrated significantly improved local control rates at 1 year ( 100 vs $58 \%, \mathrm{p}=0.06)$ [15]. Kim et al. also reported a retrospective comparison of single versus hypofractionated SRS (6 Gy $\times 6$ fractions). Despite hypofractionated SRS being used for larger tumors ( $5.0 \mathrm{vs} 2.2 \mathrm{ml}$ ) or tumors in unfavorable locations, local control was equivalent as compared with single fraction SRS (71 vs 69\%, p = 0.13). However, radiation toxicity was greater in patients treated with single fraction treatment (17 vs 5\%, p = 0.05) [16].

More recently, Minniti et al. [17] reported a retrospective comparative analysis between single fraction SRS and hypofractionated SRS $(3 \times 9$ Gy) for large brain metastases (defined as a maximum diameter of at least $2 \mathrm{~cm}$ ). This study has the strengths of very robust clinical and imaging follow-up and assessment for RN. Important findings were improved local control with use of hypofractionated SRS at 1 year as compared with single fraction SRS (91 vs $77 \%, \mathrm{p}=0.01$, respectively). Furthermore was a decreased 1-year cumulative incidence rate of radionecrosis with use of hypofractionated SRS as compared with single fraction SRS (9 vs $18 \%, p=0.01$, respectively). It is important to note that this study has defined large brain metastases as $>2 \mathrm{~cm}$, which is in keeping with historic patterns of worse local control following single fraction SRS, and our own clinical guideline to now hypofractionate these larger metastases.

Modern radiation oncology and SRS apparatus have adapted to support the growing trend of hypofractionated SRS. Further research is necessary to determine the optimal total dose and number of fractions, in addition to the treatment schedule (daily, alternate days, weekly), that maximizes local control at an acceptable rate of radiation toxicity. In addition, research is required to determine optimal dosimetric guidelines for tumor control and toxicity avoidance. Our position is that the current evidence supports hypofractionated treatment as a viable option for metastases $>2 \mathrm{~cm}$ in size or those adjacent to critical structures; however, we acknowledge that there are no randomized trials comparing single fraction SRS to hypofractionated SRS to clarify and confirm the role of each modality. 
Financial \& competing interests disclosure A Sahgal has received honoraria for past educational seminars from Medtronic and Elekta $A B$ and research grants from Elekta $A B$. The authors have no other relevant affiliations or financial involvement with any organization or

\section{References}

1 Leksell L. The stereotaxic method and radiosurgery of the brain. Acta. Chir. Scand. 102(4), 316-319 (1951).

2 Andrews DW, Scott CB, Sperduto PW et al. Whole brain radiation therapy with or without stereotactic radiosurgery boost for patients with one to three brain metastases: Phase III results of the RTOG 9508 randomised trial. Lancet 363(9422), 1665-1672 (2004).

3 Aoyama $\mathrm{H}$, Shirato $\mathrm{H}$, Tago M et al. Stereotactic radiosurgery plus whole-brain radiation therapy vs stereotactic radiosurgery alone for treatment of brain metastases: a randomized controlled trial. JAMA 295(21), 2483-2491 (2006).

4 Chang EL, Wefel JS, Hess KR et al. Neurocognition in patients with brain metastases treated with radiosurgery or radiosurgery plus whole-brain irradiation: a randomised controlled trial. Lancet Oncol. 10(11), 1037-1044 (2009).

5 Kocher M, Soffietti R, Abacioglu U et al. Adjuvant whole-brain radiotherapy versus observation after radiosurgery or surgical resection of one to three cerebral metastases: results of the EORTC 22952-26001 study. J. Clin. Oncol. 29(2), 134-141 (2011).

6 Sahgal A, Aoyama H, Kocher M et al. Phase 3 trials of stereotactic radiosurgery with or

entity with a financial interest in or financial conflict with

the subject matter or materials discussed in the manuscript

apart from those disclosed.

No writing assistance was utilized in the production of

this manuscript.

without whole-brain radiation therapy for 1 to 4 brain metastases: individual patient data meta-analysis. Int. J. Radiat. Oncol. Biol. Phys. 91(4) 710-717 (2015).

7 Tsao M, Xu W, Sahgal A. A meta-analysis evaluating stereotactic radiosurgery, whole-brain radiotherapy, or both for patients presenting with a limited number of brain metastases. Cancer 118(9), 2486-2493 (2012).

8 Soffietti R, Kocher M, Abacioglu UM et al. A European Organisation for Research and Treatment of Cancer Phase III trial of adjuvant whole-brain radiotherapy versus observation in patients with one to three brain metastases from solid tumors after surgical resection or radiosurgery: quality-oflife results. J. Clin. Oncol. 31(1), 65-72 (2013).

9 Shaw E, Scott C, Souhami L et al. Single dose radiosurgical treatment of recurrent previously irradiated primary brain tumors and brain metastases: final report of RTOG protocol 90-05. Int. J. Radiat. Oncol. Biol. Phys. 47(2), 291-298 (2000).

10 Blonigen BJ, Steinmetz RD, Levin L, Lamba MA, Warnick RE, Breneman JC. Irradiated volume as a predictor of brain radionecrosis after linear accelerator stereotactic radiosurgery. Int. J. Radiat. Oncol. Biol. Phys. 77(4), 996-1001 (2010).
11 Vogelbaum MA, Angelov L, Lee SY, Li L, Barnett GH, Suh JH. Local control of brain metastases by stereotactic radiosurgery in relation to dose to the tumor margin. J. Neurosurg. 104(6), 907-912 (2006).

12 Sahgal A, Ma L, Chang E et al. Advances in technology for intracranial stereotactic radiosurgery. Technol. Cancer Res. Treat. 8(4), 271280 (2009).

13 Nahum AE. The radiobiology of hypofractionation. Clin. Oncol. (R. Coll. Radiol.) 27(5), 260-269 (2015).

14 Kolesnick R, Fuks Z. Radiation and ceramide-induced apoptosis. Oncogene 22(37), 5897-5906 (2003).

15 Feuvret L, Vinchon S, Martin V et al. Stereotactic radiotherapy for large solitary brain metastases. Cancer Radiother. 18(2), 97-106 (2014).

16 Kim YJ, Cho KH, Kim JY et al. Single-dose versus fractionated stereotactic radiotherapy for brain metastases. Int. J. Radiat. Oncol. Biol. Phys. 81(2), 483-489 (2011).

17 Minniti, G, Scaringi C, Paolini S et al. Single-fraction versus multi-fraction $(3 \times 9$ Gy) stereotactic radiosurgery for large $(<2 \mathrm{~cm})$ brain metastases: a comparative analysis of local control and risk of radiation-induced brain necrosis. Int. J. Radiat. Oncol. Biol. Phys. pii: S0360-3016(16)00325-00324 (2016). 